\title{
AUS ZWEI MACH EINS - UND WIEDER ZURÜCK! DIE RESTAURIERUNG EINES PASTICCIO AUS DER SAMMLUNG DES JOSEPH-FERDINAND VON ÖSTERREICH-TOSKANA
}

\author{
Alexander Gatzsche ${ }^{1}$
}

\begin{abstract}
The Osiris statuette P 6169 of the Prague Náprstek Museum has a very unusual appearance: In addition to a striking surface corrosion, its crooked shape in comparison to its fine surface details are not corresponding to the ancient Egyptian artistic ideal. Through consideration of comparative pieces of the Joseph Ferdinand of Austria-Tuscany collection came up the suspicion that it might be concerned as a pasticcio. The particular challenge of the object is to establish mainly the certainty that it was possibly composed of different ancient fragments in modern times. Objectives of the restoration project are, firstly, the identification of P 6169 as a pasticcio and, secondly, a conception of conservation and restoration treatments, which have to be derived from this knowledge. In addition to the scientific and cultural-historical analysis of the object, the focus of the work is mainly on the ethical discussion on how to approach such an object and the resulting demands of the object to its preservation for the future.
\end{abstract}

KEYWORDS: pasticcio - Osiris - bronze - statuette - restoration - conservation - forgery

\section{Einführung}

Im Jahr 2013 sollte im tschechischen Olomouc eine Sonderausstellung mit dem Titel Poklady starého Egypta (Schätze des Alten Ägyptens) entstehen. Im Zuge dessen wurden verschiedene Objekte konservatorisch begutachtet. Dabei wurde eine Statuette des ägyptischen Osiris (Inventarnummer P 6196) [Taf. 1] ausgewählt, die für Archäologen und Restauratoren ein ungewöhnliches Erscheinungsbild aufwies, um diese restauratorisch zu analysieren und zu bearbeiten. Die folgenden Arbeiten lieferten dann ein wesentlich umfangreicheres Bild, was nicht nur an der Freilegung der Oberfläche lag, die weitere Details zum Vorschein brachte, sondern auch tiefere Einblicke in die modernen Überarbeitungen pfiffiger Antikenverkäufer zur Zeit der Jahrhundertwende brachte, die die heutigen Bearbeiter zu komplexen restaurierungsethischen Entscheidungen drängten.

1 Kontaktdaten: Alexander Gatzsche, AMRICHA gemeinnützige GmbH, Leipzig, Deutschland; e-mail: alexander.gatzsche@amricha.com. Dieses Werk wurde vom Kulturministerium der Tschechischen Republik finanziell unterstützt (DKRVO 2019-2023/18.III.b, Nationalmuseum, 00023272). Das im Artikel vorgestellte Projekt wurde im Rahmen der Bachelor-Arbeit „Identifizierung eines Pasticcio und Konzeptentwicklung zu dessen Konservierung und Restaurierung - eine altägyptische Osiris-Statuette aus der Sammlung Joseph-Ferdinand von Österreich-Toskana“ 2013 and der Hochschule für Technik und Wirtschaft (HTW) Berlin durchgeführt. 1. Betreuer: Prof. Dr. Kay Kohlmeyer, Berlin und 2. Betreuer: PhDr. Pavel Onderka, Prag.

This work is licensed under the Creative Commons Attribution-Noncommercial-No Devivs 3.0 License 


\section{Objektbeschreibung und -herkunft}

Die Statuette des ägyptischen Gottes Osiris war etwa $34 \mathrm{~cm}$ hoch und an seiner breitesten Stelle (Ellenbogen) etwa $8 \mathrm{~cm}$ breit und bis zu $4 \mathrm{~cm}$ dick. Osiris wurde hier mit überkreuzten Armen und mit Zeptern in den Händen darstellt. Die Krone stellte die weiße konisch nach Oben verjüngende Atef-Krone dar, die wiederum eine senkrechte Uräusschlange trug. Die seitlichen Hörner und Federn der Krone waren wie auch der Kopf der Schlange nicht mehr vorhanden, wobei sich die ehemaligen Zapflöcher dieser Bestandteile noch identifizieren ließen. Die linke Hand hielt den stilistischen Wedel und die rechte Hand das Heckar-Zepter. Dabei lagen die beiden Enden des Wedels auf dem rechten Arm auf, wogegen der Bogen des Heckar-Zepters bereits auf der linken Schulter endete. Die Bronzefigur wurde in einem Hohlgussverfahren hergestellt und später ziseliert nachgearbeitet, um Details im Gesicht und Oberkörper hauszuarbeiten.

Die Osiris-Statuette P 6169 datiert in die Spätzeit des alten Ägypten (ca. 747-332 v.Chr.). Bekannt ist, dass diese Statuette Teil einer umfangreichen Sammlung von Antiquitäten war, welche 1903 durch den Erzherzog Joseph Ferdinand von Österreich-Toskana in Olmütz (heute Olomouc) angelegt wurde. ${ }^{2}$ In den Unterlagen fanden sich mehrere Exponate die aus dem Kairoer Ägyptischen Museum in seine Sammlung übergingen. Ob auch dieses Stück dazu gehörte, ist allerdings nicht gesichert. In Olomouc etablierte der Erzherzog im Jahr 1906 das Erzherzog-Joseph-Ferdinand-Museum welches bis zur Unabhängigkeit der Tschechoslowakei (1918) Bestand hatte. Die Sammlung wurde im Zuge der Unabhängigkeit in den Besitz des tschechoslowakischen Staates übernommen (Inventarnummer A 6196). 1981 wurde die gesamte Sammlung schließlich vom Náprstek-Museum für asiatische, afrikanische und amerikanische Kulturen des Nationalmuseums Prag übernommen ${ }^{3}$ und die Osiris-Statuette bekam die Inventarnummer P 6169.

\section{Zustandsbeschreibung [Taf. 2]}

Ungewöhnlich an der Bronzestatue waren vor allem die sehr groben mit Sand vermischten Korrosionsprodukte auf der Oberfläche. Diese drohten in vielen Bereichen der Statue abzufallen, was eine unbedingte Konservierung des Objektes erforderte, um es in seinem Zustand zu erhalten. Außerdem wurden durch dieses Gemisch viele Details der Figur überdeckt und teilweise komplett unkenntlich gemacht. Weiterhin zeigte das Stück eine ungewöhnliche Verkrümmung von den Beinen zum Oberkörper hin, sodass die Statue immer gefährdet gewesen war umzufallen. Das ist deswegen so ungewöhnlich, weil die Standfläche am unteren Ende eindeutig auf die Fixierung auf einem Sockel hinwies. Demnach war die Statuette dereinst stehen und nicht liegend konzipiert.

Es war bereits zum Zeitpunkt der Begutachtung vor der Restaurierung deutlich zu erkennen, dass das Innere der Figur mit Gips ausgegossen war, was eine ungewöhnliche Massivität zur Folge hatte. Darüber hinaus führte der sehr hygroskopische Gips dazu, dass sich an den Bereichen wo die Figur offen war (also in diesem Fall unter den Beinen) bereits starke, aktive türkisgrüne Kupferchlorid-Korrosion gebildet hatte. ${ }^{4}$ Es war daher zu erwarten, dass sich ein ähnliches Korrosionsbild im Inneren der Statue vorfinden musste, was eine aktive Gefährdung des Objektes darstellte. Auf der Oberfläche befan-

2 Mynářová et. al. 2013, S. 103.

3 Mynářová et. al. 2013, S. 104.

4 Peltz 2009a, S. 206. 
den sich dazu noch mehrere teilweise historische Labels mit alten Inventarnummern und auf dem Sockel, in weißer Tusche aufgebracht, die aktuelle Inventarnummer P 6196.

\section{Naturwissenschaftliche Analysen}

Um sich Gewissheit über den Zustand der Figur zu verschaffen, wurden umfangreiche optische und naturwissenschaftliche Analysen durchgeführt. Zu Beginn wurden mikroskopische Untersuchungen unternommen. Dabei zeigte sich recht bald, dass die als Korrosion bezeichnete Oberfläche mit mehreren organischen Bestandteilen durchsetzt war, was an sich ungewöhnlich für eine natürlich gewachsene Korrosion ist, außer das Objekt besaß selbst im Originalzustand eine Hülle aus einem solchen Material. Bei der Betrachtung unter UV-Licht zeigte sich dann, dass das gesamte Objekt lumineszierte [Taf. 3], was auf ein organisches Bindemittel zurückzuführen ist. Im Zuge dessen wurden Proben von der Oberfläche entnommen und diese im Querschliff betrachtet, als auch mikrochemischen Analysen unterzogen, um das Bindemittel zu bestimmen.

Die mikroskopische Betrachtung des Querschliffs zeigte eine ungewöhnliche Struktur im Querschnitt. Insgesamt konnten drei Schichten körnigen Materials (wahrscheinlich Sand), durchsetzt mit grüner Kupferkorrosion und organischen Fasern identifiziert werden [Taf. 4]. Dies sprach eindeutig gegen eine natürlich gewachsene Korrosion. Die mikrochemischen Analysen ergaben eine positive Testung auf Proteine und auch auf Kohlehydrate, allerdings ein negatives Ergebnis auf Schwefel und damit Eigelb. ${ }^{5}$ Da das positive Ergebnis auf Kohlenhydrate auch auf die pflanzlichen Fasern im Gefüge zurückgeführt werden kann, bleiben noch die Proteine als Grundlage für den verwendeten Klebstoff. Als Bindemittel ist demnach ein tierischer Leim zu identifizieren, der das Gefüge aus Sand und Korrosion zusammengehalten hat. Dieses wurde in mindestens drei Schichten auf die Oberfläche des Objektes aufgebracht.

Um das innere Gefüge der Figur beurteilen zu können wurden außerdem mehrere Röntgenaufnahmen angefertigt, die weiteren Aufschluss über den Zustand des Objektes lieferten [Taf. 5]. ${ }^{6}$ Es zeigte sich eindeutig, dass die Figur aus mindestens drei Teilen zusammengesetzt war. Dabei glichen sich die Hüft-bis-Bein-Partie mit den Füßen der Figur in ihrer materiellen Struktur und hoben sich deutlich vom eher blasig erscheinen Teil des Oberkörpers ab. Im Inneren befanden sich anscheinend noch zwei materiell sehr kompakte Nägel, die wohl in den später eingeführten Gips eingelassen wurden, um als zusätzliche Armierung der drei Teile zu dienen. Dass beide Nägel so kompakt im Röntgenbild erschienen, lässt darauf schließen, dass sie entgegen der Erscheinung des gesamten Objektes nicht stark korrodiert waren [Taf. 6].

Bei der genaueren Betrachtung der Röntgenbilder, vor allem des Oberkörpers, konnten zusätzliche Details erkannt werden, welche so auf der Figur bisher nicht sichtbar waren. So zeigten sich klar erkennbare Verzierungen und stilistische Details, die von der zuvor ermittelten Masse aus Sand, Korrosion und Bindemittel überdeckt waren. Neben den ziselierten Augen und Brauen waren auch Ohren zu erkennen und eine deutliche Trennung von der Krone zum Gesicht. Hinzu kommt, dass noch ein eingelassener Zapfen des ehemals vorhandenen Bartes des Osiris vorhanden war.

5 Die Vorgehensweise bei der mikrochemischen Analyse folgte dabei der Publikation von Schramm und Hering 2000.

6 Durchgeführt wurden die Röntgenaufnahmen an einem dem Studiengang der Konservierung und Restaurierung der HTW Berlin eigenen Röntgengeräts. 


\section{Identifizierung als Pasticcio und weitere Osiris-Pasticci in der Sammlung des Náprestek Museums}

Die zuvor erlangten Erkenntnisse aus den naturwissenschaftlichen Analysen verwiesen darauf, dass es sich bei der Osiris-Statue P 6196 um ein so genanntes Pasticcio handelt (Pasticcio = ital. "Gemisch" "Durcheinander" , plural = Pasticci). Das heißt also ein aus mehreren Fragmenten von unterschiedlichen Figuren zusammen gesetztes Objekt. ${ }^{7}$

Die Intention, solche Objekte zu fertigen wandelte sich mit der Zeit. In der damals noch jungen Ägyptologie kann als einer der berühmtesten „Produzenten“ solcher Pasticci Karl-Richard Lepsius nachgewiesen werden. Nachdem er mehrere Objekte von seiner Reise nach Ägypten und in den Sudan mitbrachte, wurden diese bei ihrer Aufstellung im frisch errichteten Neuen Museum umfassend „restauriert“. Dazu gehörten sowohl die Ergänzung von fehlenden Köpfen, Rekonstruktion ganzer Statuen anhand einer erhaltenen Basis oder eben das Zusammenführen mehrerer unterschiedlicher Originalfragmente. ${ }^{8}$

Lepsius verfolgte allerdings nicht die Intention der gezielten Täuschung, sondern verfolgte damit andere Ziele. Der Gesamteindruck sollte dazu führen, dass „... die einzelnen unzusammenhängenden Denkmäler dieser fern liegenden Kulturepoche ein leichteres Verständnis und eine größere Lebendigkeit für den Beschauer erhalten...". 9 Es ging Lepsius also vor allem darum, den Besuchern der Sammlung einen Gesamteindruck zu vermitteln, welcher seiner Meinung nach mit einzelnen Fragmenten wohl nicht zu erreichen gewesen wäre. Wenn man also bedenkt, dass die Ägyptologie zu diesem Zeitpunkt noch recht jung war, müssen Lepsius Intentionen in diesem Kontext betrachtet werden, auch wenn sie unseren heutigen restaurierungsethischen Vorstellungen bei Weitem nicht mehr entsprechen.

Anders verhält es sich mit der Prager Bronzefigur P 6196. Zu Zeiten, als der Erzherzog Joseph-Ferdinand von Österreich-Toskana dieses Objekt erwarb, war in ganz Europa bereits das Fieber der "Ägyptomanie“ ausgebrochen. Die Nachfrage an antiken ägyptischen Objekten bei Reisenden nach Ägypten war groß und mit vollständigen Objekten ließ sich mehr Geld verdienen, als mit einzelnen Fragmenten. Dass bei der Zusammensetzung solcher Objekte oft nachlässig gearbeitet wurde, zeigt sich vor allem auch an der hier besprochenen Osiris-Figur. Der Wert am Objekt bemaß sich demnach vor allem in seiner Vollständigkeit und weniger an den hochwertigen Details der Einzelteile eines solchen Pasticcio. Um den Gesamteindruck zu vereinheitlichen wurden solche Figuren mit der oben bereits besprochenen künstlichen „Insitu-Patina“ überzogen, die einen fundfrischen Zustand simulieren sollte. ${ }^{10}$

Dass so eine Zusammenführung und Überarbeitung auch zu teilweise grotesken Erscheinungen führen konnte, zeigt sich an anderen Figuren der Sammlung des Erzherzog Joseph-Ferdinand von Österreich-Toskana. ${ }^{11}$ So befinden sich in der Sammlung des Náprstek-Museums noch drei weitere große Osiris-Figuren aus Bronze die aus der Sammlung des Erzherzogs stammen: P 6194, P 6195 und P 6346. Alle sind circa $30 \mathrm{~cm}$ groß und zwei von ihnen (P 6194 und P 6346) weisen deutliche Hinweise auf, dass es

$7 \quad$ Peltz 2009b, S. 73.

8 Mehlitz 2011, S. 205; Peltz und Zorn 2009, S. 247.

9 Lepsius 1880, S. 3.

10 Peltz und Zorn 2009, S. 247.

11 Mynářová et. al. 2013, S. 73, Abb. 7. 
sich auch bei ihnen um Pasticci handeln könnte. P 6194 zum Beispiel zeigt eine vollkommen unterschiedliche Korrosionserscheinung des Kopfes im Vergleich zum restlichen Körper und P 6348 besitzt einen eindeutig viel zu kleinen Kopf im Vergleich zum Körper. Auch der Osiris P 6195 weist unterschiedliche Korrosionserscheinungen an Kopf und Körper auf, wenn auch nicht so deutlich wie bei den anderen Stücken. Allerdings ist der Übergang vom Hals zum Kopf ungewöhnlich steif und gleicht einem Stehkragen, was ein weiteres Argument für ein Pasticcio sein dürfte. ${ }^{12}$

\section{Restaurierungsethische Überlegungen im Umgang mit dem Pasticcio}

Aufgrund der Ergebnisse der Untersuchungen zum Objekt P 6196 musste folglich eine Entscheidung über die weiteren Maßnahmen getroffen werden.

Die Untersuchungen zum Objekt P 6196 offenbarten ein Pasticcio aus drei Fragmenten von mindestens zwei Statuetten als ein Befund, der die weiteren konservatorischen und restauratorischen Maßnahmen nachhaltig beeinflussen sollte. Eine ähnliche Vorgehensweise fand sich auch bei anderen Stücken gleichen Typs in der Sammlung. An der Außenoberfläche zeigte die oberste Schichte des aus Sand und Kupferkorrosion bestehenden Überzuges aufgrund des Austrocknens des tierischen Leimes eine schollenartige Ablösung. Insbesondere aber durch die hygroskopischen Eigenschaften der Gipsfüllung im Inneren und der dadurch ausgelösten Aktivierung inaktiver Chlorid-Korrosion stellte das Objekt in seinem Zustand eine Gefährdung für sich selbst dar. Um das Objekte im aktuellen Zustand zu erhalten, hätten umfangreiche Festigungsmaßnahmen und eine nur durch Absperrung der oberen Schichten erfolgende Entsalzung stattfinden müssen. Letztere wäre allerdings aufgrund der kompakten Erscheinung des Objektes in ihrem Erfolg ungewiss gewesen.

Zur Debatte standen darüber hinaus eine Auflösung des Pasticcio und eine damit verbundene Lesbarmachung seiner Einzelteile. Da es sich dabei aber um einen enormen Eingriff in die Originalsubstan $z^{13}$ handelte, hätte eine besondere Sorgfalt auf die Dokumentation des Objektes gelegt werden müssen.

Grundsätzlich war die Osiris-Figur unter Betrachtung seiner vollständigen Geschichte zu beurteilen. Demnach handelte es sich in ihrem Zustand als Pasticcio um eine eigenständige Objektkategorie. Die Entscheidung jedoch fiel am Ende auf die Auflösung des Pasticcio. Die wichtigsten Gründe dafür lagen vor allem in der Selbstgefährdung des Objektes in seinem aktuellen Zustand, und dem gegenüberstehenden hohen Aufwand mit ungewissem Erfolg, wenn man es als Pasticcio hätte erhalten wollen. Gleichzeitig war diese Maßnahme deswegen zu vertreten, da andere gleichartige Pasticci in der Sammlung weiterhin vorhanden wären und die Auflösung von einem Exemplar einen wissenschaftlichen Mehrgewinn versprach. Dieser lag zum einen in der Offenlegung der Konstruktion des Pasticcio und zum anderen in der Sichtbarmachung von stilistischen Details, die eine weitere Herkunftsanalyse der Einzelfragmente möglich machen sollte.

12 Eine deutlich umfangreichere Studie zum „Phänomen“ des Passticio in der Museumslandschaft im Hinblick auf dessen Umfang und seiner Variationen ist sicher ein erstrebenswertes Projekt. Dessen (Teil-) Integration in diesen Artikel, hätte jedoch seinen Umfang bei Weitem gesprengt, den gelegten Fokus deutlich verlagert und trotzdem hätte die Thematik nicht ausreichend behandelt werden können.

13 Als Originalsubstanz wird hier das Material bezeichnet welches bei Beginn der Restaurierung zum Objekt gehörte. Das Pasticcio an sich ist das Originalobjekt welches aus mehreren Bestandteilen zusammengesetzt wurde. Der Begriff ist daher von den antiken Bestandteilen zu unterscheiden. 


\section{Restauratorische Maßnahmen}

Nachdem sich für die Auflösung des Pasticcio in seine historischen Einzelteile entschieden wurde, musste anhand der vorliegenden Informationen eine fragmentschonende Vorgehensweise entwickelt werden. Zuerst sollten die Einzelteile der Figur getrennt und anschließend separat bearbeitet werden. Dazu wurden mögliche Eintrittspunkte in die Figur anhand der Röntgenbilder lokalisiert [Taf. 8].

Um den Zustand der Osiris-Statuette P 6196 in seiner Form als Pasticcio vor den restauratorischen Eingriffen bestmöglich zu dokumentieren, wurden neben den angefertigten Fotografien zusätzlich noch digitale 3D-Scans mittels eines Streifenlichtprojektionsscanners ${ }^{14}$ angefertigt, wodurch das Objekt zumindest virtuell in seiner Form erhalten blieb [Taf. 9].

Die anschließende Trennung der Einzelteile des Pasticcio erfolgte sowohl mechanisch, als auch mit rotierenden Schleifkörpern. Am schwierigsten war dabei die Trennung der beiden oberen Teile. Die als Armierung verwendeten Eisennägel im Inneren des Pasticcio ermöglichten es nur schrittweise die beiden Teile voneinander zu trennen. Die Oberflächen der dann separat vorliegenden Fragmente wurden anschließend unter dem Mikroskop Schritt für Schritt mechanisch sowohl an der Außen- und Innenoberfläche von nicht dazu gehörigem Material freigelegt.

Nach der Freilegung zeigte sich auch deutlich der Unterschied des Oberkörpers zu den beiden unteren Fragmenten in seiner anderen Oberflächenerscheinung [Taf. 10-11]. Dieser Unterschied wurde anschließend mit der Röntgenfluoreszenzanalyse ${ }^{15}$ und der hierdurch aufgezeigten abweichenden Legierungszusammensetzung der Fragmente bestätigt [Tab. 1]. Die Unterteile weisen im Vergleich zum Oberteil Zink als Legierungsbestandteil auf. Demnach gehören die beiden unteren Teile vermutlich zusammen und der Oberkörper stammt von einer anderen Statuette.

Tab. 1. Elementverteilung auf der Basis der RFA-Analysen. Dafür wurden die Analysen im Inneren der Fragmente an blanken Stellen vorgenommen.

\begin{tabular}{|c|c|c|c|c|c|c|}
\hline Element & Oberteil & Error in & Unterteil Bauch & Error in & Unterteil Füße & Error in \\
\hline $\mathrm{Fe}$ & $0,16 \%$ & 0,03 & $0,76 \%$ & 0,07 & $0,84 \%$ & 0,08 \\
\hline $\mathrm{Ni}$ & $0,31 \%$ & 0,05 & $-^{*}$ & - & $-^{*}$ & - \\
\hline $\mathrm{Cu}$ & $91,67 \%$ & 4,54 & $80,84 \%$ & 3,54 & $78,62 \%$ & 3,91 \\
\hline $\mathrm{Zn}$ & $0,01 \% * *$ & 0,10 & $1,23 \%$ & 0,14 & $1,20 \%$ & 0,16 \\
\hline $\mathrm{Sn}$ & $2,79 \%$ & 0,29 & $4,89 \%$ & 0,40 & $4,75 \%$ & 0,44 \\
\hline $\mathrm{Pb}$ & $5,01 \%$ & 0,33 & $11,45 \%$ & 0,58 & $13,76 \%$ & 0,76 \\
\hline $\mathrm{Sb}$ & $0,05 \% * *$ & 0,06 & $0,82 \%$ & 0,15 & $0,83 \%$ & 0,17 \\
\hline
\end{tabular}

* Nickel konnte im Spektrum nicht identifiziert werden.

** Diese Werte werden vom Error-Faktor überstiegen. Daher ist anzunehmen, dass dieses Element nicht mit messbaren Werten in der Legierung vorhanden ist.

14 Zur Anwendung des hier benutzten Gerätes in der Restaurierung siehe Gatzsche 2014.

15 Auch Röntgenfluoreszenzspektroskopie - zur ausführlichen Beschreibung der technologischen Grundlagen siehe Römpp 1995, S. 3902. 
Daneben wurden auch noch die Bestandteile des noch vorhandenen Bartzapfens untersucht. ${ }^{16}$ Dabei zeigte sich ein deutlicher materieller Unterschied durch einen erhöhten Bleiwert im Vergleich zum Oberkörper. Das führte sicherlich auch zu einer farblichen Differenzierung des Bartes gegenüber dem Körper, welche heute aufgrund der Korrosion nicht mehr so deutlich zu Tage tritt.

Als überraschend stellte sich der Detailreichtum des Oberteils heraus, welcher in den Röntgenbildern zuvor nur zu erahnen war. Im Brust- und Nackenbereich konnte ein nahezu vollständig erhaltener viergliedriger Halskragen freigelegt werden [Taf. 12]. Dieser besteht aus vier übereinander liegenden umlaufenden Ringen an dessen unterer Ring noch eine weitere Reihe von tropfenförmigen Perlen anschließt. Der Halskragen wurde nach dem Guss in die Objektoberfläche geschnitten, was bei den Ringen Fehlziselierungen bezeugen [Taf. 13].

Neben der Ziselierung des eigenständigen Kragens finden sich weitere Oberflächendetails auf dem linken Zepter, wo sich beim oberen Ansatz ein Rautenmuster nach dem Guss ergänzt wurde [Taf. 14]. Weiterhin besitzt der Schlangenkörper einen vom Kopfansatz zum oberen Ende durchlaufenden positiv aufragender Mittelstrich zu dessen beide Seiten Punkt-Ziselierungen vorgenommen wurden, um den Körper des Tieres eine natürliche Musterung zu geben [Taf. 15].

Außerdem konnten bei der vorsichtigen Freilegung auch Reste einer ehemaligen Vergoldung im Gesicht und im Kragenbereich gesichert werden [Taf.16]. Das Ergebnis einer anschließend durchgeführten Röntgenfluorenzanalyse zeigt, dass es sich hier um ein sehr reines Gold mit geringem Silberanteil handelt. ${ }^{17} \mathrm{Um}$ welche Art der technischen Vergoldung es sich hierbei handelt, kann aufgrund der geringen Erhaltung dieser Bereiche nicht abschließend geklärt werden. Die Figur trug demnach mehrere Tauschierungen, wohl aus Edelmetallen, und zumindest bei den Zeptern vielleicht auch Einlagen aus Edelmetallen oder Glas. Im Inneren des Oberteils konnten sogar noch Reste des Gusskerns gesichert werden [Taf. 17], der bei der Herstellung im Hohlgussverfahren noch in Überresten im Inneren verblieb.

Nach Abschluss der Arbeiten wurden alle Teile, auch die Armierungsnägel und das abgenommene Material, als Proben zusammen mit den Statuettenfragmenten in einer Lagerungs- und Transportverpackung sicher verpackt und so wieder dem Depot zugeführt [Taf. 18].

\section{Ausblick}

Die Figur befindet sich nun wieder im Prager Náprstek-Museum und kann dort sicher verwahrt und zukünftig ausgestellt werden. So wurde bei der Folgeausstellung der 2013er Ausstellung das Stück P 6196 zusammen mit seiner Verpackung und allen Proben und entnommenen Einzelteilen ausgestellt und mit den noch zusammengehörigen Pasticci gezeigt. In dieser Form ist es nun möglich, dem Besucher neben den kulturhistorischen Informationen solcher Osiris-Statuetten auch die sammlungs- und restaurierungsgeschichtlichen Aspekte der vermeintlich vollständigen Statuette darzulegen.

16 Die Messung des Bartzapfens erfolgte lediglich graphisch und nicht wie bei den anderen Messungen aufgeschlüsselt nach Elementkonzentrationen, wodurch diese Messung in ihren prozentualen Werten nicht der Tab. 1 beigefügt werden konnten. In dieser Messung zeigte sich jedoch als dominierendes Material Blei als eindeutig.

17 Prozentuale Kalkulation des Spektrums: Au 97,43 \% Error 2,05; Ag 2,57 \% Error 0,15. 


\section{Literatur:}

Gatzsche, A. (2014). Low-Cost but High Quality, Eine kostengünstige 3-D-Dokumentationsmethode, in Restauro. Zeitschrift für Restaurierung, Denkmalpflege und Museumstechnik, Ausgabe 8, München: Verlag Georg D. W. Callwey, S. 22-27.

Lepsius, K. R. (1880). Beschreibung der Wandgemälde in der Ägyptischen Abtheilung, 4. Auflage. Berlin: Königliche Museen zu Berlin.

Mehlitz, H. (2011). Richard Lepsius, Ägypten und die Ordnung der Wissenschaft. Berlin: Kulturverlag Kadmos Berlin.

Mynářová, J., Onderka, P., Podhorný, R. and Vrtal, V. (2013). Poklady Starého Egypta, Sbírka Josefa Ferdinanda Habsburského. Olomouc: Vlastivědné muzeum v Olomouci.

Peltz, U. (2009a). Der Originalität auf der Spur, Die Ent- und Neurestaurierung der Bronzegefäße von Boscoreale. In: U. Peltz und O. Zorn, kulturGUTerhalten, Restaurierung archäologischer Schätze an den Staatlichen Museen zu Berlin. Berlin: Phillip von Zabern, S. 206-207.

Peltz, U. (2009b). Oberflächenvielfalt, Standards früherer Restaurierungen antiker Bronzen der Berliner Antikensammlung. In: U. Peltz und O. Zorn, kulturGUTerhalten, Standards in der Restaurierungswissenschaft und Denkmalpflege. Berlin: Phillip von Zabern, S. 71-81.

Peltz, U. und Zorn, O. (2009). Hauptsache schön?! - Die perfekten „Originale“, Pasticci, Fälschungen und Kopien. In: U. Peltz und O. Zorn, kulturGUTerhalten, Restaurierung archäologischer Schätze an den Staatlichen Museen zu Berlin. Berlin: Phillip von Zabern, S. 246-247.

Römpp, H., Begr., Falbe, J. und Regitz, M., Hrsg. (1995). Römpp Chemie Lexikon. Stuttgart - New York: Georg Thieme Verlag.

Schramm, H.-P. und Hering, B. (2000). Historische Malmaterialien und ihre Identifizierung. Berlin: Ravensburger Buchverlag. 


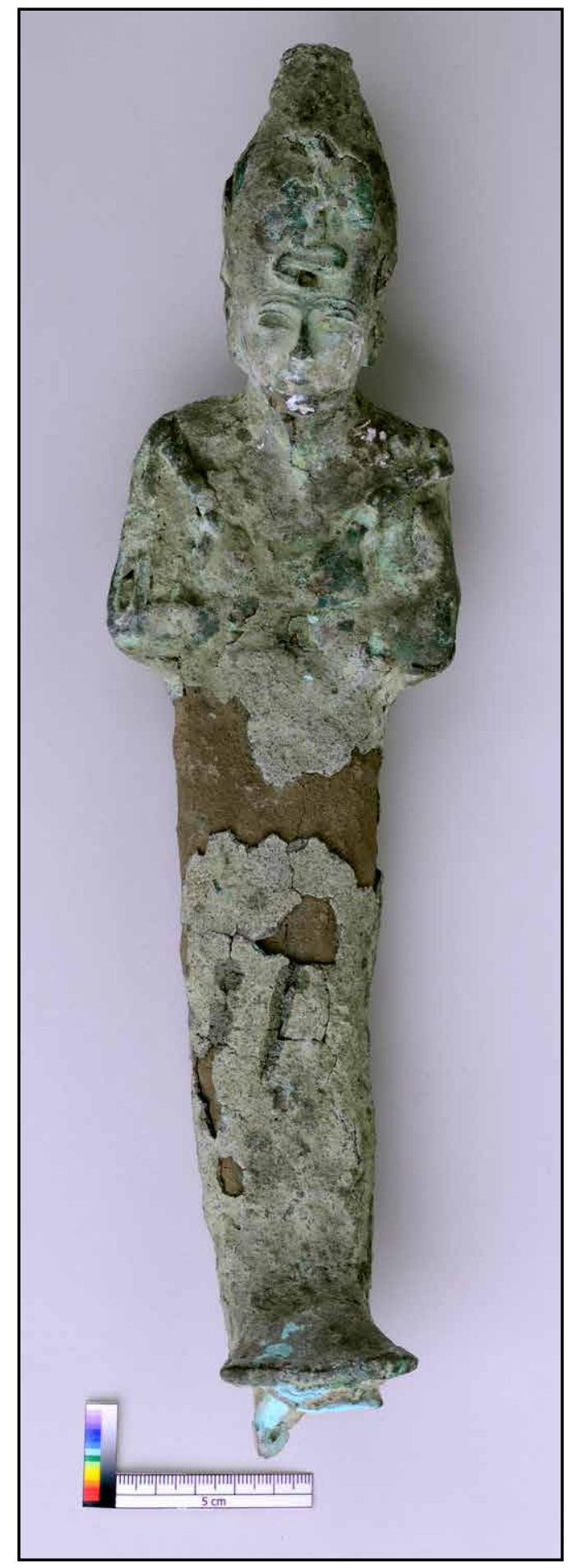

Taf. 1. Osiris-Statuette P 6196 vor der Restaurierung. 


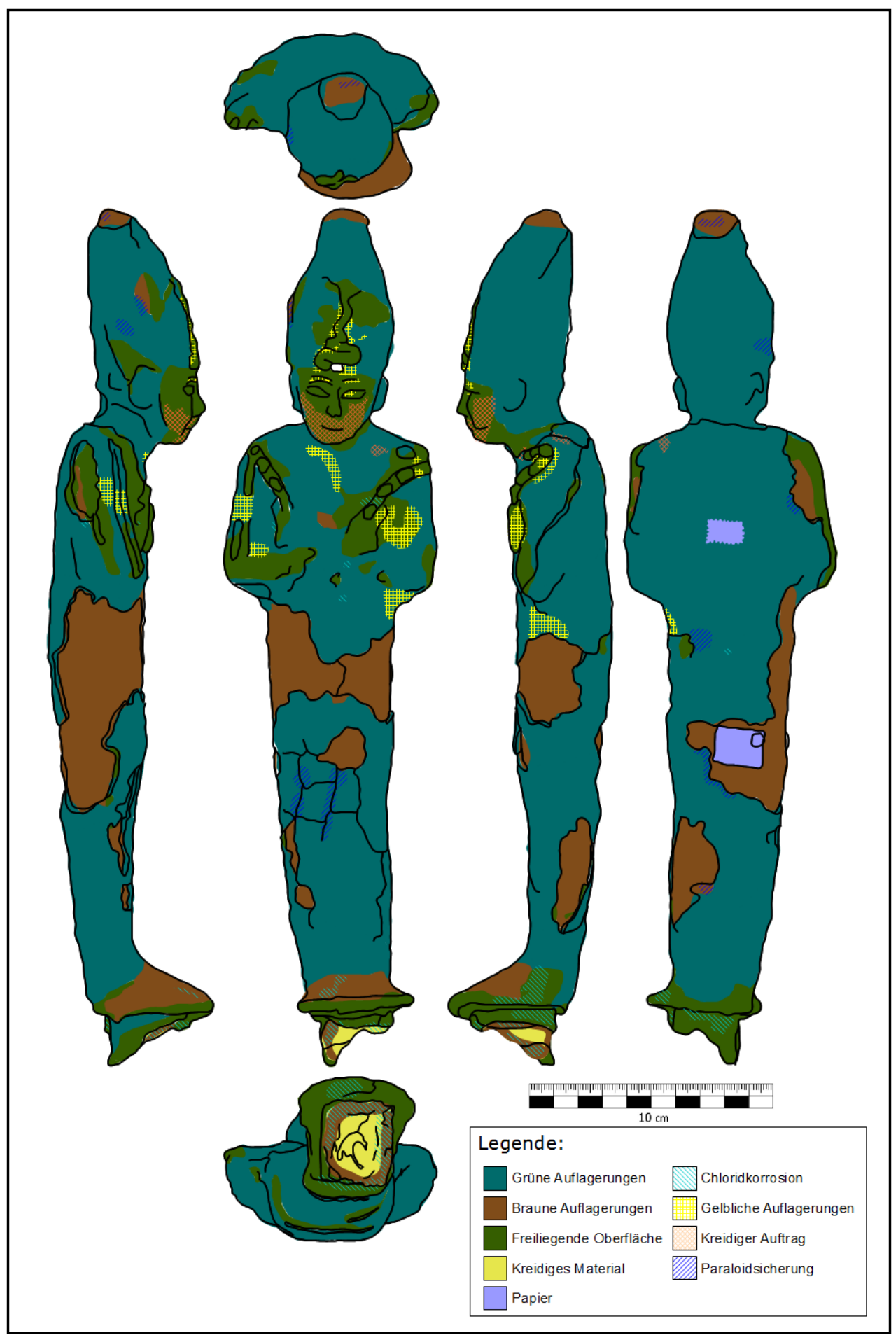

Taf. 2. Zustandskartierung anhand äußerer Betrachtung. 


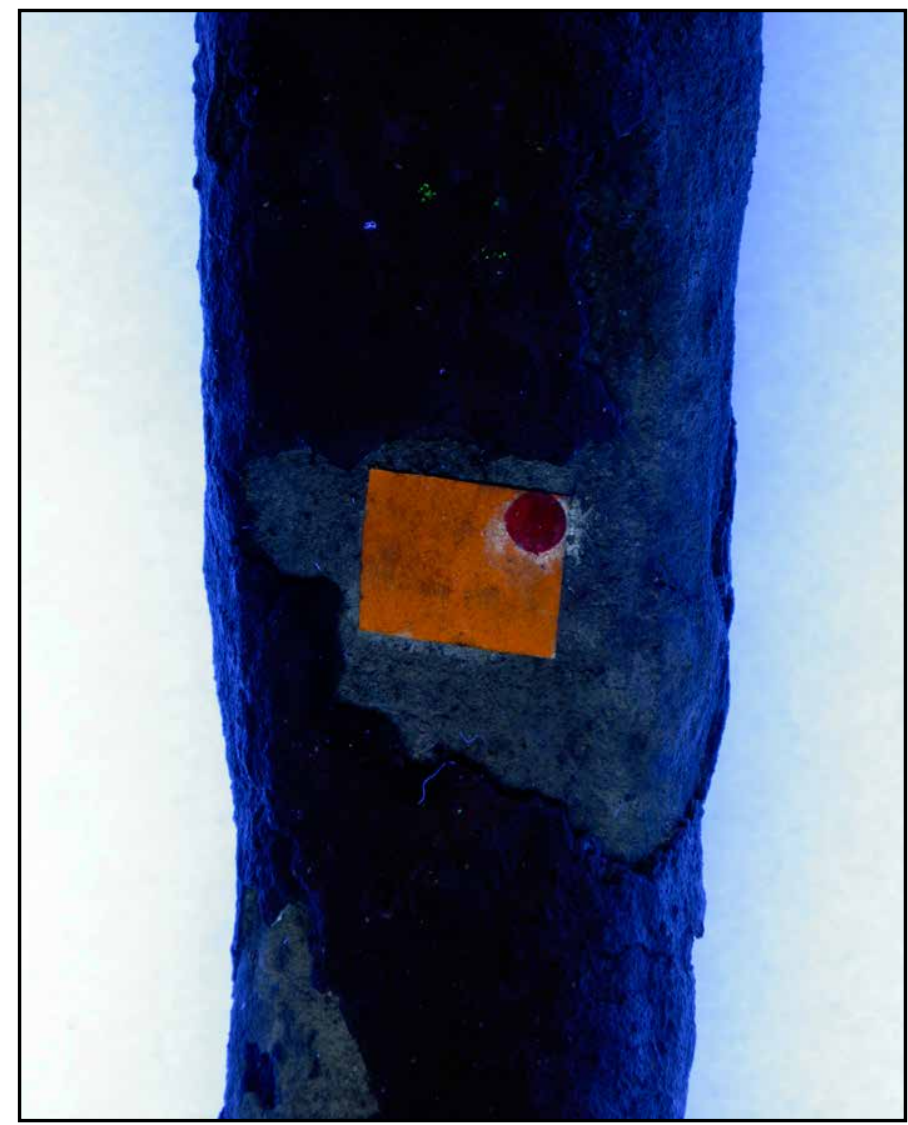

号

Taf. 3. Fluoreszenz der Oberfläche unter Anregung mit ultraviolettem Licht.

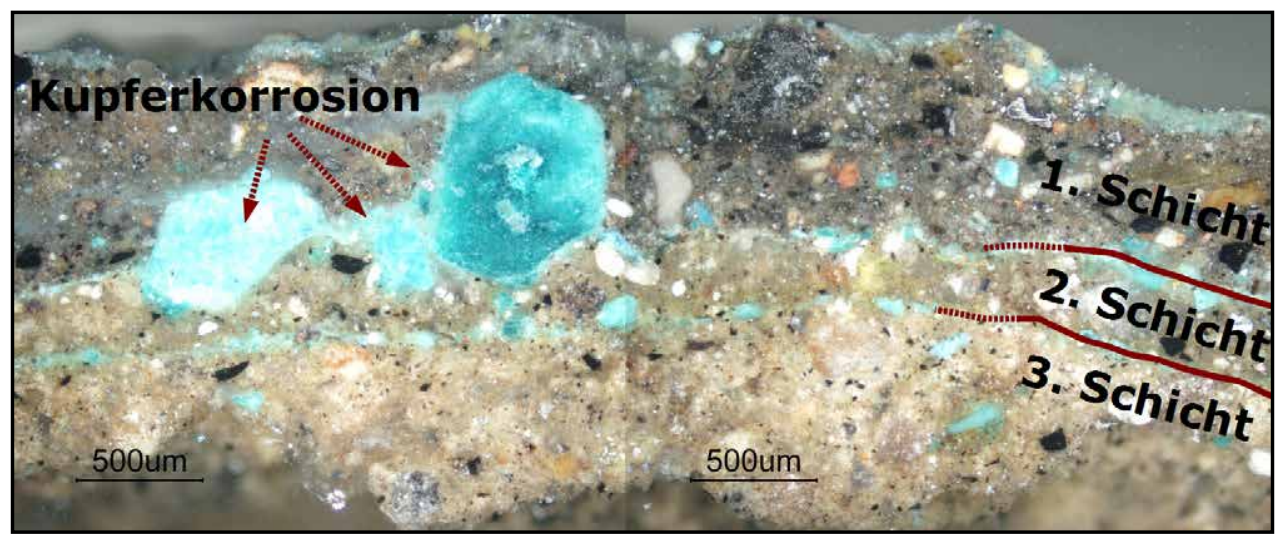

Taf. 4. Querschliff der künstlich aufgetragenen Masse aus Kupferkorrosion und Sand. 


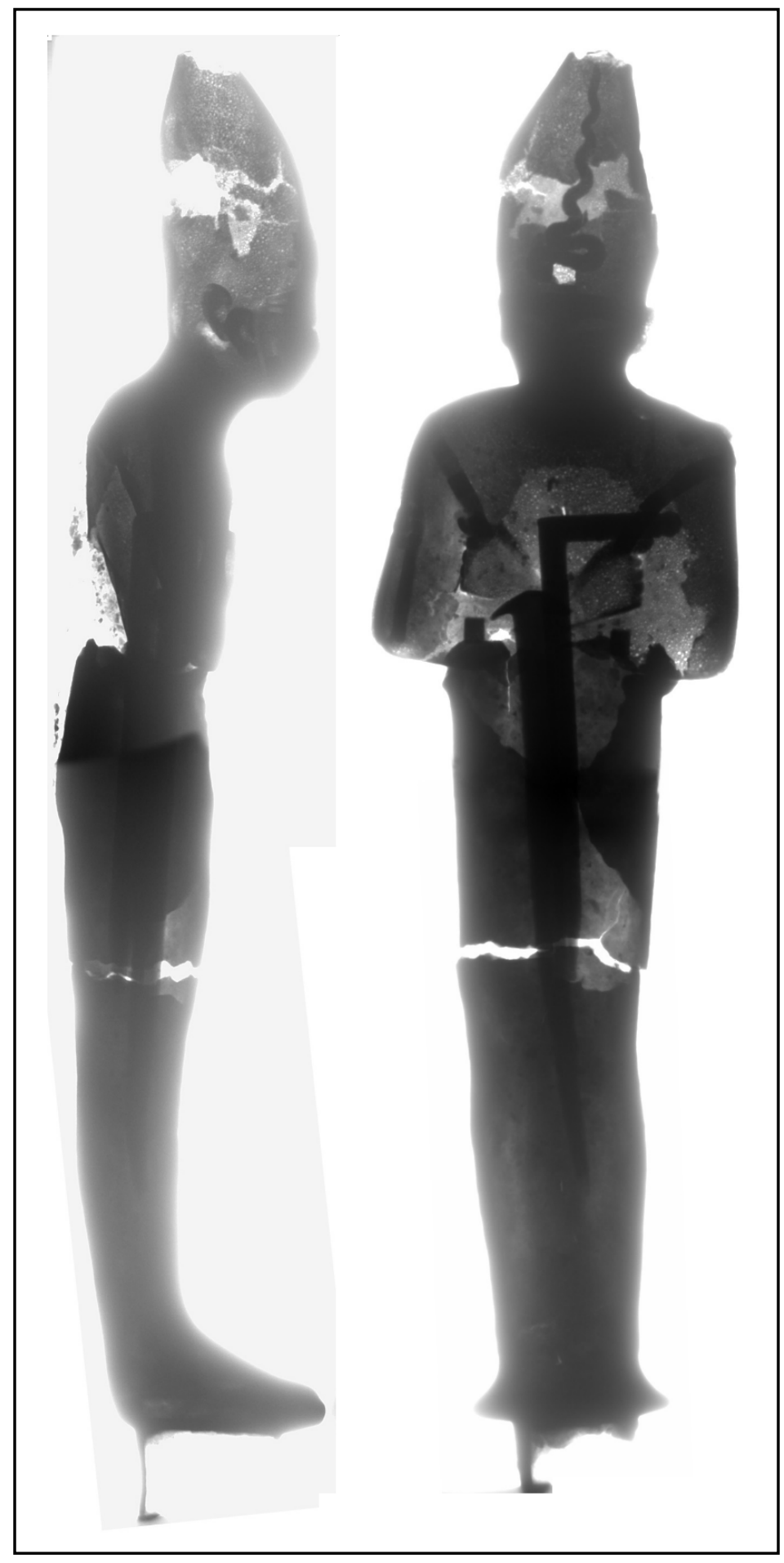

Taf. 5. Frontale und seitliche Röntgenaufnahmen des Objektes. 


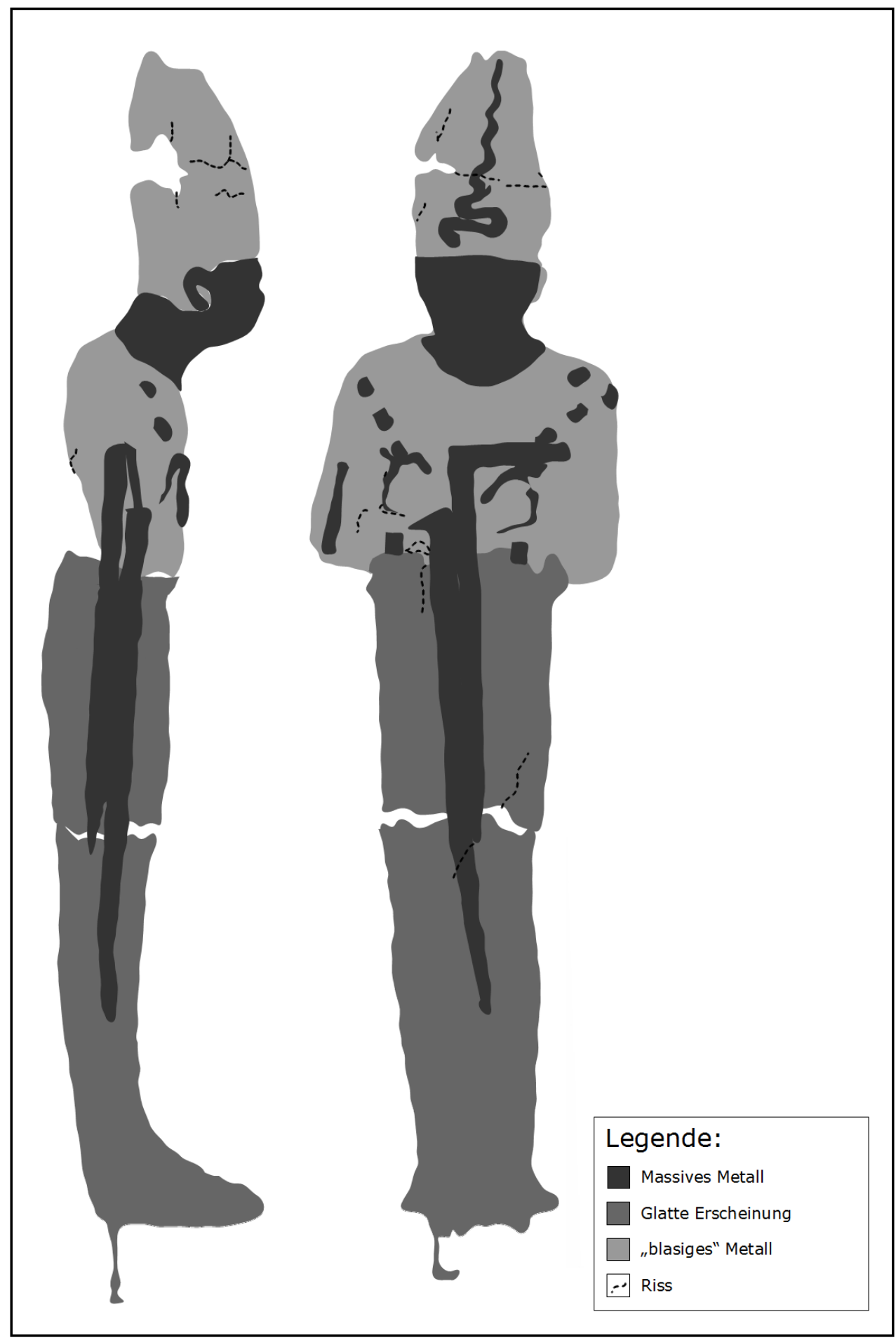

Taf. 6. Kartierung der Röntgenaufnahmen zur Visualisierung. 


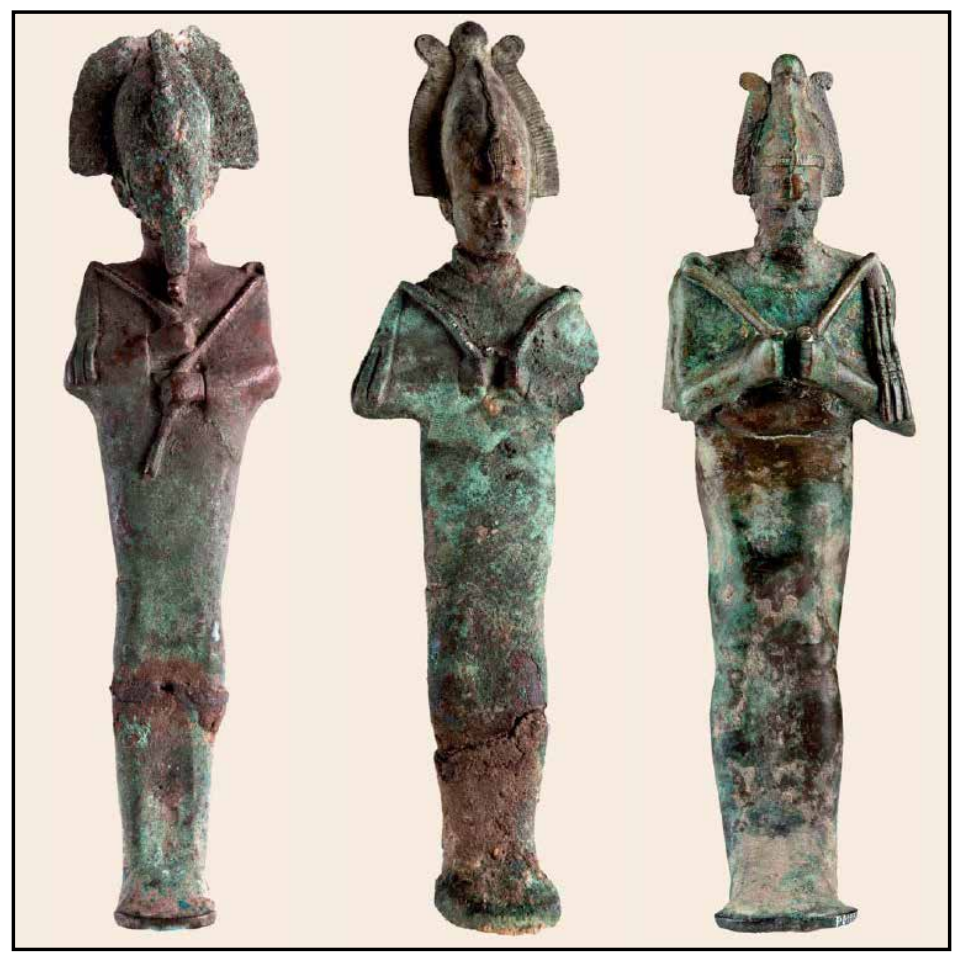

Taf. 7. Weitere Osiris-Statuetten aus der Sammlung des Erzherzogs - v.l.n.r. P 6194, P 6195 und P 6346.

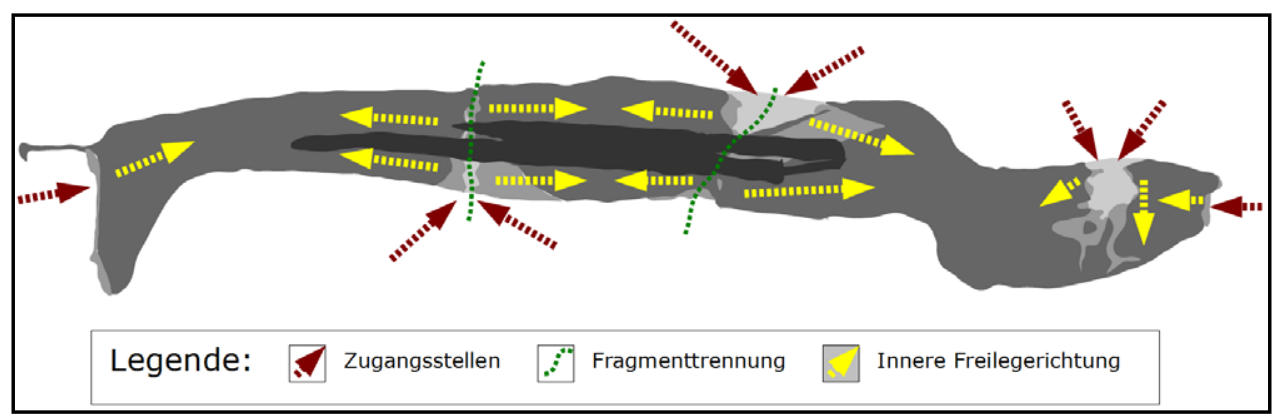

Taf. 8. Anhand der Röntgenbilder geplante Vorgehensweise bei der Trennung und Freilegung der einzelnen Fragmente. 



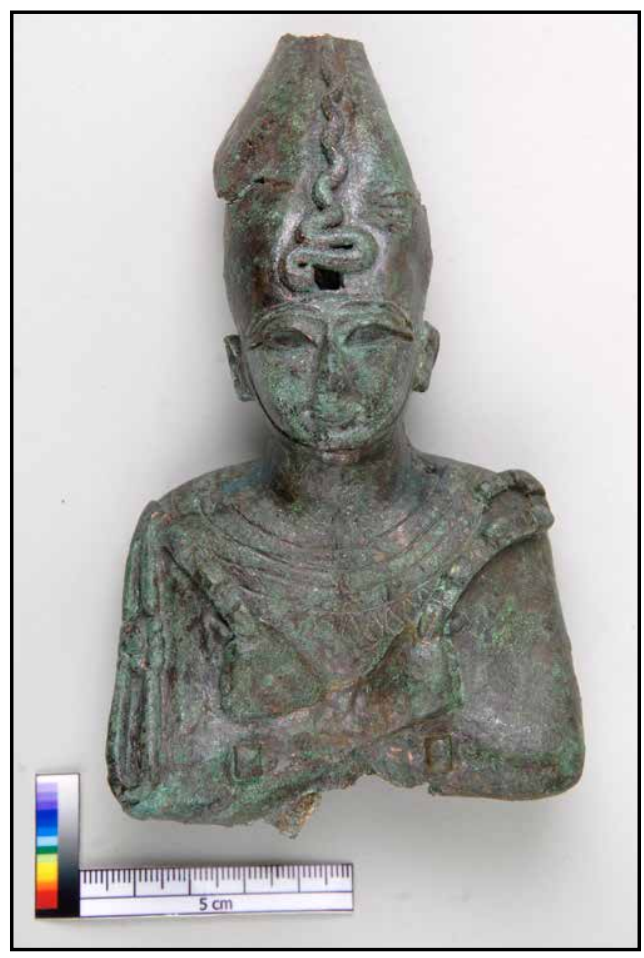

Taf. 10. Oberes Fragment nach der Restaurierung.

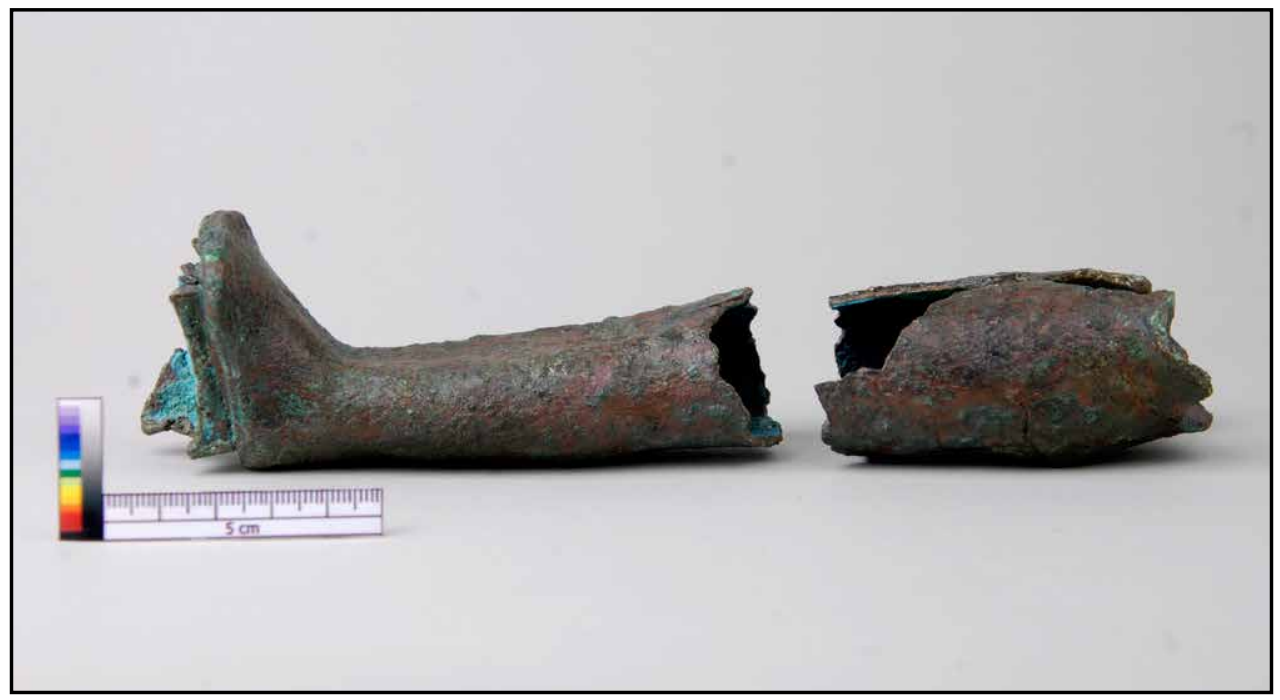

Taf. 11. Untere Fragmente nach der Restaurierung. 


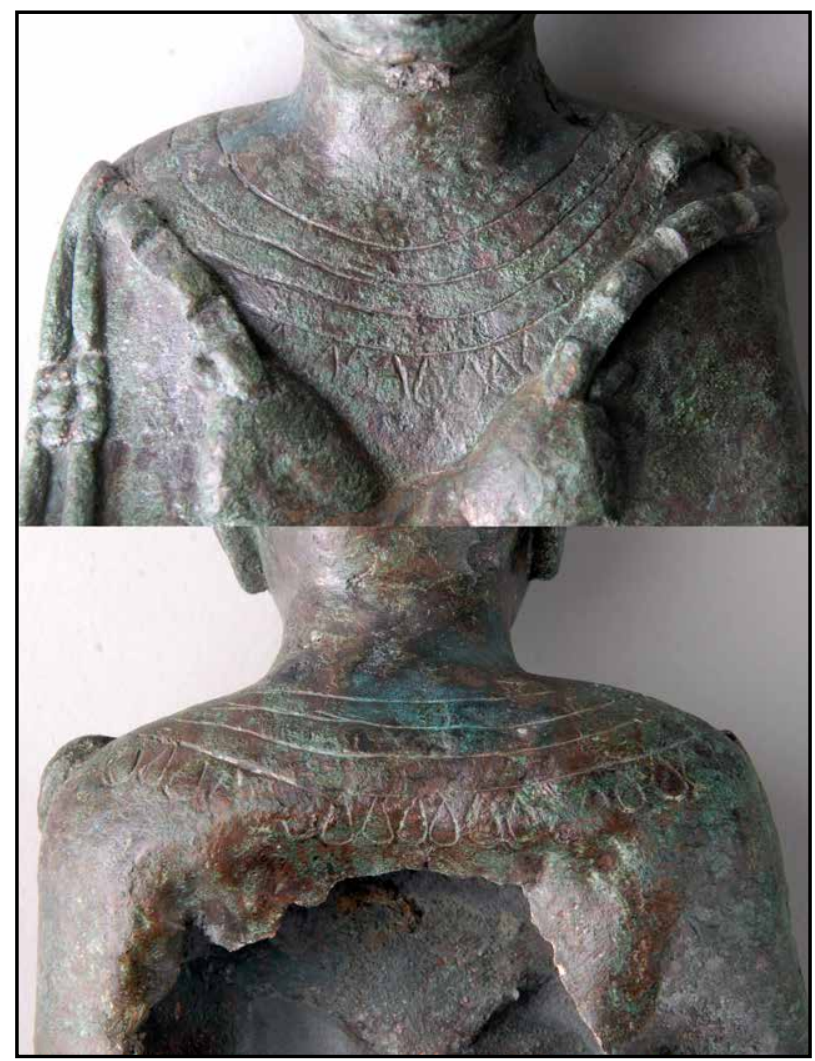

品

Taf. 12. Freigelegter Halskragen mit ziselierten Tropfenperlen.

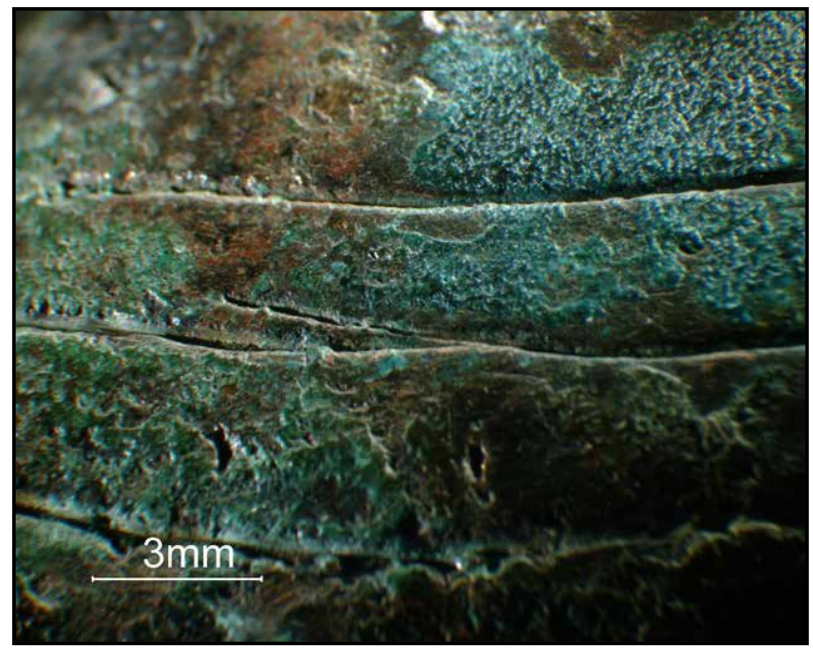

Taf. 13. auslaufender "Schnitzer“ der Linienziselierung auf der Schulter, ein Fehler bei der Nachbearbeitung. 


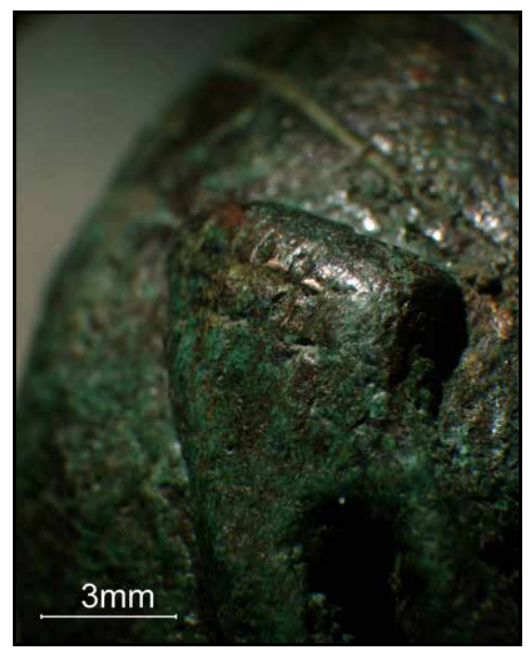

Taf. 14. Rautenmuster auf dem oberen Knick des linken Wedels auf der Brust des Osiris.

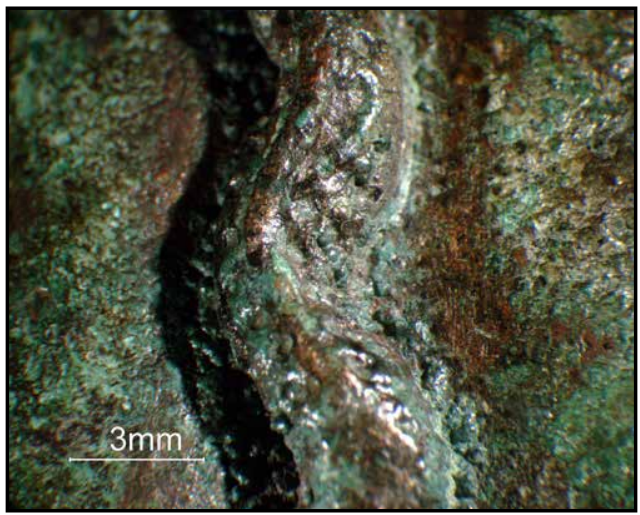

Taf. 15. Punkt- und Strichziselierung auf dem Schlangenkörper zur Schuppenimitation.

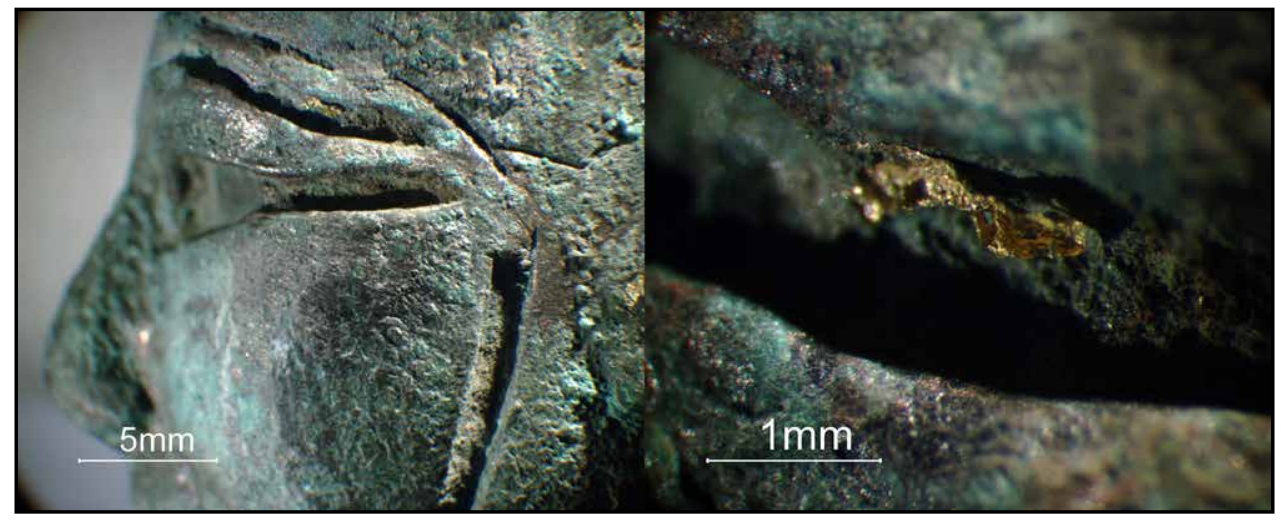

Taf. 16. Mikroskopisches Detail der Ziselierungen im Gesichtsbereich mit Resten von Vergoldung (Detail der Augenbaue). 


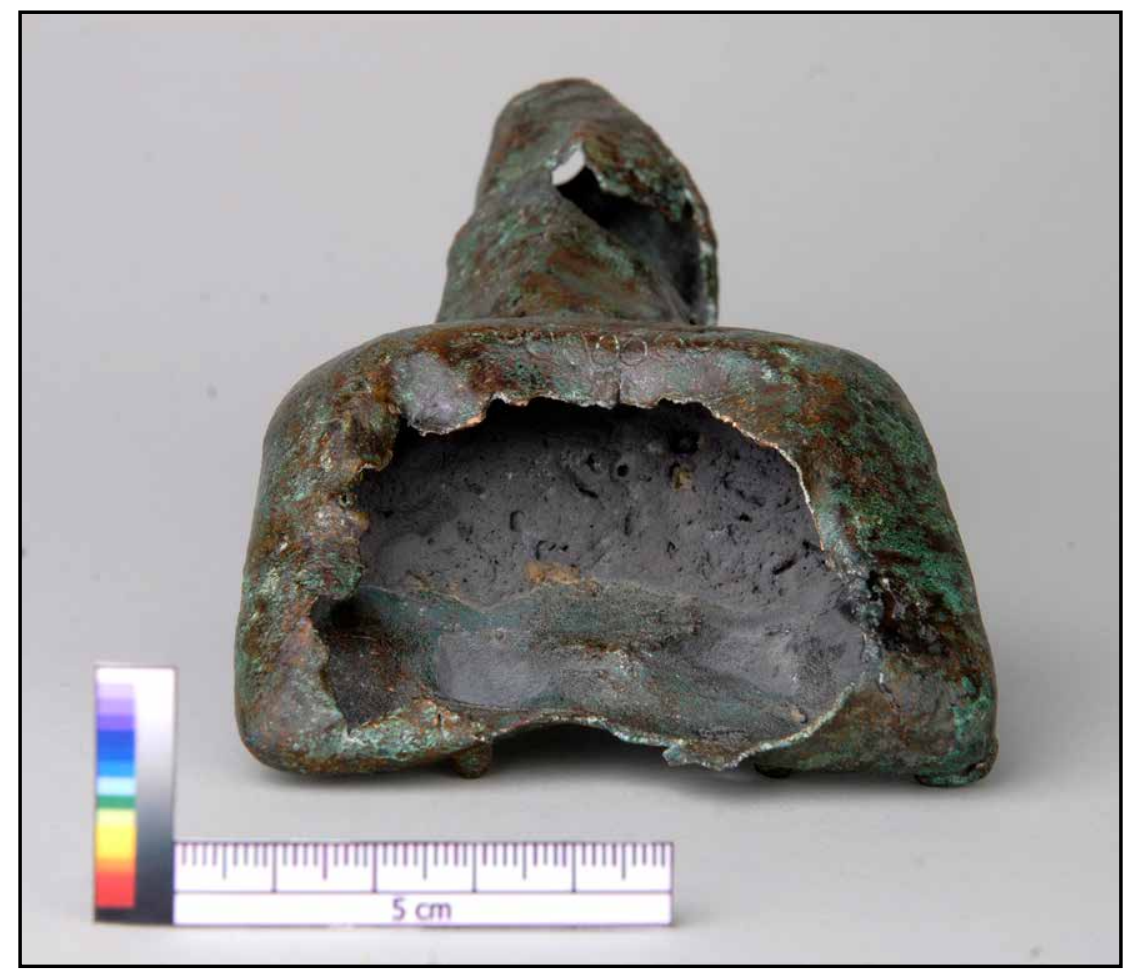

品

Taf. 17. Reste des antiken Gusskerns im Inneren des Oberteils.

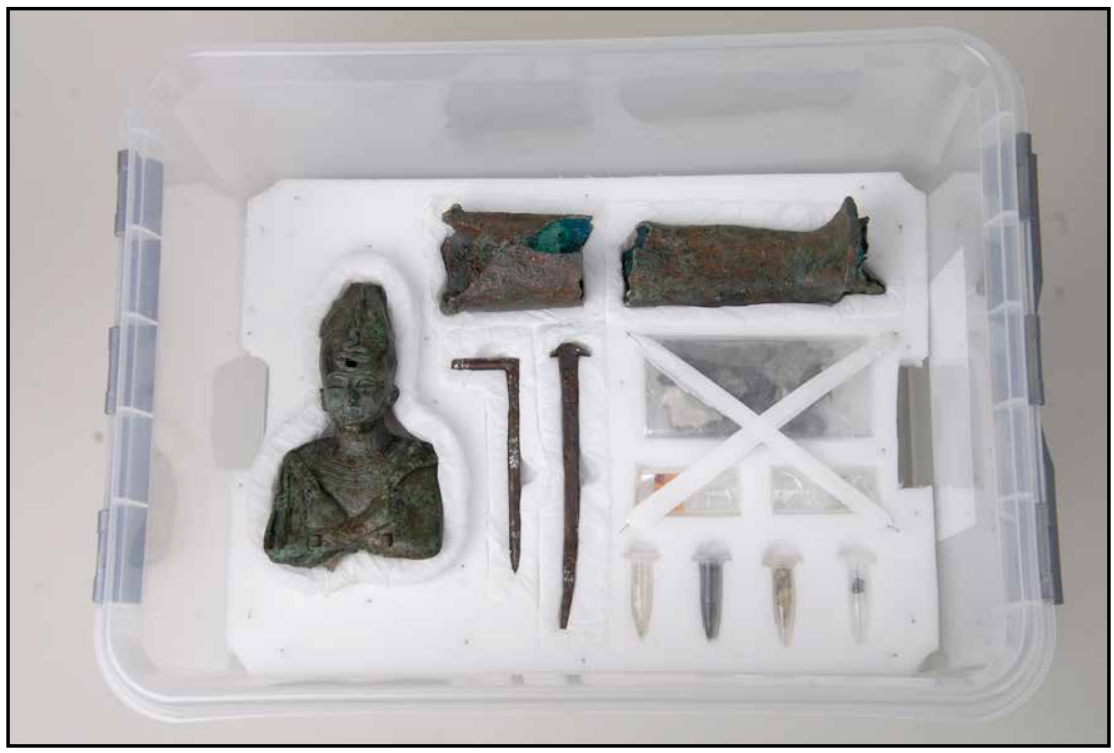

Taf. 18. Verpackung aller Fragmente zusammen mit den Armierungsnägeln und Proben der entnommenen Materialien. 